BULL. AUSTRAL. MATH. SOC.

VOL. $29(1984), 99-110$.

\title{
PERIODIC SOLUTIONS OF THE BOUNDARY VALUE PROBLEM FOR THE NONLINEAR HEAT EQUATION
}

\author{
M. N. NKashama AND M. WILLEM
}

We prove the existence of generalized periodic solutions of the boundary value problem for the nonlinear heat equation. The proof is based on classical Leray-Schauder's techniques and coincidence degree.

\section{Introduction}

Let $J=[0,2 \pi] \times[0, \pi]$ and let $H=L^{2}(J)$ be the space of measurable Lebesgue square integrable real functions on $J$ with the usual inner product $(.,$.$) and corresponding norm |$.$| . Suppose that h \in H$ and $g: J \times R \rightarrow R$ is a function such that $g(, \ldots, u)$ is measurable on $J$ for each $u \in R, g(t, x,$.$) is continuous on R$ for a.e. $(t, x) \in J$. We shall then say that $g$ satisfies Caratheodory conditions. Moreover we suppose that $g$ satisfies a linear growth condition, i.e. there exists a constant $c>0$ and a real valued function $d \in H$ such that $|g(t, x, u)| \leqslant c|u|+d(t, x)$ for all $u \in R$ and a.e. $(t, x) \in J$.

Consider the problem

(H)

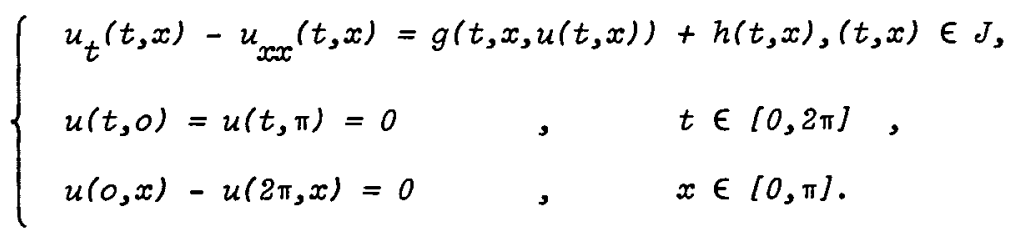

We shall prove existence results for $(H)$ under nonuniform nonresonance conditions.

Received 17 February 1984.

Copyright Clearance Centre, Inc. Serial-fee code: 0004-9727.84 $\$ A 2.00+0.00$ 
Let

$$
\begin{gathered}
H^{1}(J)=\left\{u \in H: u_{t}, u_{x} \in H\right\} \text { and } \\
H^{1,2}(J)=\left\{u \in H^{1}(J): u_{x x} \in H\right\} \text { with }
\end{gathered}
$$

respectively

$$
|u|_{1}^{2}=\int_{0}^{2 \pi} \int_{0}^{\pi}\left(u^{2}(t, x)+u_{t}^{2}(t, x)+u_{x}^{2}(t, x)\right) d x d t
$$

and

$$
|u|_{1,2}^{2}=\int_{0}^{2 \pi} \int_{0}^{\pi}\left(u^{2}(t, x)+u_{t}^{2}(t, x)+u_{x}^{2}(t, x)+u_{x x}^{2}(t, x)\right) d x d t .
$$

$H^{1}(J)$ and $H^{1,2}(J)$ are Banach spaces with these norms. Denote by $H_{0}^{1}(J)$ the closure in $H^{1}(J)$ of all real functions $u(t, x)$ on $J$ which are infinitely continuously differentiable such that

$$
\begin{array}{ll}
u(t, 0)=u(t, \pi)=0, & t \in[0,2 \pi], \\
u(0, x)-u(2 \pi, x)=0, & x \in[0, \pi] .
\end{array}
$$

A generalized periodic solution to the problem (H) is a function $u \in H^{1,2}(J) \cap H_{0}^{1}(J)$ which satisfies the equation $\left(H_{1}\right)$ a.e. on $J$. In particular, the periodic-Dirichlet problem on $J$ for the nonhomogeneous linear equation

$$
u_{t}(t, x)-u_{x x}(t, x)-\lambda u(t, x)=h(t, x)
$$

is uniquely solvable for every $h \in H$ if and only if

$$
\lambda \neq m^{2}, m \in \boldsymbol{N}^{*},
$$

(see e.g. [6], [9] or [3]).

In [6], [9], [19] it has been proved that the problem (H) has at least a generalized periodic solution if there exists real numbers $p, q, r>0$ such that for some $m \in \boldsymbol{N}^{*}$

$$
m^{2}<p \leqslant u^{-1} g(t, x, u) \leqslant q<(m+1)^{2}
$$

for a.e. $(t, x) \in J$ and all $u \in R$ such that $|u| \geqslant r$. 
The aim of this paper is to generalize this result when $(0.2)$ is replaced by conditions of the form

$$
\begin{aligned}
m^{2} \leqslant \gamma(t, x) \leqslant \lim _{|u| \rightarrow+\infty} \text { inf } u^{-1} g(t, x, u) \leqslant & \lim _{|u| \rightarrow+\infty} \sup ^{-1} g(t, x, u) \\
& \leqslant \Gamma(t, x) \leqslant(m+1)^{2}
\end{aligned}
$$

or

$$
\lim _{|u| \rightarrow+\infty} \sup u^{-1} g(t, x, u) \leqslant \Gamma(t, x) \leqslant 1
$$

for some real functions $\gamma, \Gamma$ with some supplementary conditions on the interaction of $\gamma$ and $\Gamma$ with $m^{2}$ and $(m+1)^{2}$ [or 1] respectively (see Section 1 for details). Both results are based on Leray-Schauder's type techniques and coincidence degree (see e.g. [10]). Conditions of the form $(0.3)$ or $(0.4)$ have been considered recently by many authors, namely by Berestycki and De Figueiredo [2], Gossez [7], Mawhin, Ward [12], [14], Mawhin, Ward and one of the authors [15], Iannacci and one of the authors [8] and others for ordinary, delay differential equations, elliptic partial differential equations and wave equation.

Define the linear operator

$$
\begin{aligned}
L: & \operatorname{Dom} L \subset H \rightarrow H \text { by } \\
& \operatorname{Dom} L=H_{0}^{1}(J) \cap H^{1,2}(J) \text { and } \\
& L u=u_{t}+E u \text { where } E u=-u_{x x}
\end{aligned}
$$

so that $E$ is self-adjoint and $L$ is closed, densely defined linear operator such that $\operatorname{Ker} L=(\operatorname{Im} L)^{\perp}$ and $L^{-1}$ is compact (see e.g.. [9] for details).

\section{Main results}

Suppose that $g$ satisfies Caratheodory conditions and a linear growth condition (see section 0 ). 
THEOREM 1. Assume that the inequalities

(1.1) $\quad r(t, x) \leqslant \lim _{|u|++\infty} \inf _{\mid-1} u^{-1} g(t, x, u) \leqslant \underset{|u| \rightarrow+\infty}{\lim \sup } u^{-1} g(t, x, u) \leqslant \Gamma(t, x)$

hold uniformly for a.e. $(t, x) \in J$, where $\gamma, \Gamma \in L^{\infty}(J)$ satisfy the following conditions for some $m \in N^{*}$ :

$$
m^{2} \leqslant \gamma(t, x) \leqslant \Gamma(t, x) \leqslant(m+1)^{2} \text { for a.e. }(t, x) \in J
$$

with

(1.3) $\quad a(t) \equiv \int_{0}^{\pi}\left(\gamma(t, x)-m^{2}\right) \sin ^{2} m x d x>0$ for a.e. $t \in[0,2 \pi]$

and

$b(t) \equiv \int_{0}^{\pi}\left((m+1)^{2}-\Gamma(t, x)\right) \sin ^{2}(m+1) x d x>0$ for a.e. $t \in[0,2 \pi]$

then the problem (H) has at least one GPS for each $h \in H$.

REMARK 1 . When $\gamma(t, x) \equiv \gamma(x)$ and $\Gamma(t, x) \equiv \Gamma(x)$ i.e. $\gamma$ and $\Gamma$ are independent of $t$, conditions (1.2) and (1.3) are equivalent to:

$$
m^{2} \leqslant \gamma(x) \text { and } \Gamma(x) \leqslant(m+1)^{2} \text { for a.e. } x \in[0, \pi]
$$

with strict inequalities on subsets of $[0, \pi]$ of positive measure.

To prove theorem 1 , we need some useful lemmas:

LEMMA 1.1. Let $m \in \boldsymbol{N}^{*}$ and let $p \in L^{\infty}(J)$ be such that for a.e. $(t, x) \in J, m^{2} \leqslant p(t, x) \leqslant(m+1)^{2}$ with moreover for a.e. $t \in[0,2 \pi]$,

$$
\begin{aligned}
& \int_{0}^{\pi}\left(p(t, x)-m^{2}\right) \sin ^{2} m x d x>0 \text { and } \\
& \int_{0}^{\pi}\left((m+1)^{2}-p(t, x)\right) \sin ^{2}(m+1) x d x>0
\end{aligned}
$$

then the equation

$$
\left\{\begin{array}{l}
u_{t}(t, x)-u_{x x}(t, x)-p(t, x) u(t, x)=0 \\
u(t, 0)=u(t, \pi)=0 \\
u(0, x)-u(2 \pi, x)=0
\end{array}\right.
$$

has only the trivial solution. 
Proof. The problem (1.5) is equivalent to

$$
L u-p u=0
$$

where $L$ is defined in Section 0 .

Let $u \in$ Dom $L$ be a GPS to the problem $(1.6)$, then $u$ has the Fourier series

$$
u(t, x)=\sum_{\substack{k \in \mathbb{Z}^{n} \in \mathbb{N}^{*} \\ u_{k n}}} e^{i k t} \sin n x
$$

Consider $u_{1}=\sum_{\substack{k \in \mathbb{Z} \\ n \leqslant m}} u_{k n} e^{i k t} \sin n x$ and $u_{2}=\sum_{\substack{k \in \mathbb{Z} \\ n \geqslant m+1}} u_{k n} e^{i k t} \sin n x$.

Taking into account the symmetry of $E$ and the orthogonality of $u_{1}$ and $u_{2}$, one gets easily that

$$
0=\left(u_{2}-u_{1}, L u-p u\right)=\left(E u_{2}-p u_{2}, u_{2}\right)-\left(E u_{1}-p u_{1}, u_{1}\right)
$$

Moreover by the Parseval-Steklov equality:

$$
\begin{aligned}
\left(E u_{2}-p u_{2}, u_{2}\right) & \geqslant\left(E u_{2}, u_{2}\right)-(m+1)^{2}\left(u_{2}, u_{2}\right)= \\
& =\sum_{\substack{k \in \mathbb{Z} \\
n \geqslant m+1}}\left(n^{2}-(m+1)^{2}\right)\left|u_{k n}\right|^{2} \geqslant 0
\end{aligned}
$$

and $\left(E u_{1}-p u_{1}, u_{1}\right) \leqslant \sum_{\substack{k \in \mathbb{Z} \\ n \leqslant m}}\left(n^{2}-m^{2}\right)\left|u_{k n}\right|^{2} \leqslant 0$.

Therefore (1.7) is satisfied if and only if

$$
\left(E u_{2}-p u_{2}, u_{2}\right)=0
$$

and

$$
\left(E u_{1}-p u_{1}, u_{1}\right)=0
$$

so that $u_{k n}=0$ for $k \in \mathbb{Z}$ and $n>m+1$ or $n<m$.

Hence $u_{1}=(\sin m x) \sum_{k \in \mathbb{Z}} u_{k m} e^{i k t} \equiv(\sin m x) v(t)$ 
and

$$
u_{2}=(\sin (m+1) x) \sum_{k \in \mathbb{Z}} u_{k(m+1)} e^{i k t} \equiv(\sin (m+1) x) w(t)
$$

From (1.8) and (1.9) we have

$$
\begin{aligned}
& \int_{0}^{2 \pi} \int_{0}^{\pi}\left(p(t, x)-m^{2}\right) \sin ^{2} m x d x v^{2}(t) d t=0 \text { and } \\
& \int_{0}^{2 \pi} \int_{0}^{\pi}\left((m+1)^{2}-p(t, x)\right) \sin ^{2}(m+1) x d x\left(\omega^{2}(t)\right) d t=0 .
\end{aligned}
$$

By our assumptions on $a$ and $b$ we must have $v(t)=0$ and $w(t)=0$ for a.e. $t \in[0,2 \pi]$. Thus $u_{1}=u_{2}=0$ and the proof is complete.

LEMMA 1.2. Let $\gamma, \Gamma \in L^{\infty}(J)$ be such that for a.e. $(t, x) \in J$

$$
m^{2} \leqslant \gamma(t, x) \leqslant \Gamma(t, x) \leqslant(m+1)^{2}, m \in \mathbb{N}^{*}
$$

with for a.e. $t \in[0,2 \pi]$

$$
\begin{aligned}
& \int_{0}^{\pi}\left(\gamma(t, x)-m^{2}\right) \sin ^{2} m x d x>0 \text { and } \\
& \int_{0}^{\pi}\left((m+1)^{2}-\Gamma(t, x)\right) \sin ^{2}(m+1) x d x>0
\end{aligned}
$$

then there exists $\varepsilon=\varepsilon(\gamma, \Gamma)>0$ and $\delta=\delta(\gamma, \Gamma)>0$ such that for any $p \in L^{\infty}(J)$ satisfying $\gamma(t, x)-\varepsilon \leqslant p(t, x) \leqslant \Gamma(t, x)+\varepsilon$ for a.e. $(t, x) \in J$, one has

$$
|L u-p u| \geqslant \delta|u|_{1}
$$

for all $u \in$ Dom $L$.

Proof. If it is not the case, one can find a sequence $\left(u_{n}\right)$ in Dom $L$, with $\left|u_{n}\right|_{1}=1\left(n \in \boldsymbol{N}^{*}\right)$ and sequences $\left(v_{n}\right)$ in $H,\left(p_{n}\right)$ in $L^{\infty}(J)$ such that

$$
\begin{gathered}
r(t, x)-n^{-1} \leqslant p_{n}(t, x) \leqslant \Gamma(t, x)-n^{-1} \text { for a.e. }(t, x) \in J \\
L u_{n}-p_{n} u_{n}=v_{n}, n \in \mathbb{N}^{*} \text { and } \\
v_{n} \rightarrow 0 \text { strongly in } \mathrm{H} .
\end{gathered}
$$


Using the boundedness of the sequences $\left(u_{n}\right),\left(p_{n}\right),\left(L u_{n}\right)$ in $H$, the finite dimension of Ker $L$, the compactness of $L^{-1}$ and the weak closedness of $L$, we can assume, going if necessary to subsequences that, for $n \rightarrow+\infty$,

$$
\begin{aligned}
& u_{n} \rightarrow u \text { strongly in } H_{0}^{1}(J) \\
& p_{n} \rightarrow p \text { weakly in } L^{\infty}(J)-\text { weak* } \\
& L u_{n} \rightarrow L u \text { weakly in } \mathrm{H} \text { and }|u|_{1}=1 \\
& \gamma(t, x) \leqslant p(t, x) \leqslant \Gamma(t, x) \text { for a.e. }(t, x) \in J .
\end{aligned}
$$

Now, if $\varphi \in \mathcal{C}_{o}^{\infty}(J)$, we have

$$
\begin{aligned}
\left(p_{n} u_{n}-p u, \varphi\right) & =\left(p_{n}\left(u_{n}-u\right), \varphi\right)+\left(\left(p_{n}-p\right) u, \varphi\right) \\
& \left.\leqslant c\left|u_{n}-u\right||\varphi|+\mid\left(p_{n}-p\right) u, \varphi\right) \mid .
\end{aligned}
$$

Both terms of the right hand member go to zero if $n \rightarrow+\infty$. Hence, from the density of $C_{o}^{\infty}(J)$ in $H$, we have that $p_{n} u_{n} \rightarrow p u$ weakly in $H$ when $n \rightarrow+\infty$, so that

$$
L u-p u=0 \text {. }
$$

Lemma 1.1 implies that $u=0$, a contradiction with $|u|_{1}=1$ and the proof is complete.

We are now in a position to prove Theorem 1 .

Proof of Theorem 1. Let $\varepsilon>0$ and $\delta>0$ be associated to $\gamma$ and $\Gamma$ by Lemma 1.2, then there exists a real $r=r(\varepsilon)>0$ such that for a.e. $(t, x) \in J$ and all $u \in R$ with $|u| \geqslant r$,

$$
\gamma(t, x)-\varepsilon \leqslant u^{-1} g(t, x, u) \leqslant \Gamma(t, x)+\varepsilon .
$$

The equation $(H)$ is then equivalent to

$$
u_{t}(t, x)-u_{x x}(t, x)=\tilde{\gamma}(t, x, u(t, x)) u(t, x)+f(t, x, u(t, x))+h(t, x),(t, x) \in d
$$




$$
\begin{aligned}
& u(t, 0)=u(t, \pi)=0, t \in[0,2 \pi] \\
& u(0, x)-u(2 \pi, x)=0, x \in[0, \pi]
\end{aligned}
$$

where

$$
\begin{aligned}
& \tilde{\gamma}(t, x, u)=u^{-1} g(t, x, u), \text { for } \quad|u| \geqslant r \\
& \tilde{\gamma}(t, x, u)=r^{-1} g(t, x, r) \frac{u}{r}+\left(1-\frac{u}{r}\right) \Gamma(t, x) \text {, for } 0 \leqslant u<r \\
& \tilde{\gamma}(t, x, u)=r^{-1} g(t, x,-r) \frac{u}{r}+\left(1+\frac{u}{r}\right) \Gamma(t, x), \text { for } \quad-r<u<0
\end{aligned}
$$

and

$$
f(t, x, u)=g(t, x, u)-\tilde{\gamma}(t, x, u) u
$$

The function $\tilde{\gamma}(t, x, u)$ is of Caratheodory's type since $g$ is, moreover

$$
\begin{gathered}
\gamma(t, x)-\varepsilon \leqslant \tilde{\gamma}(t, x, u) \leqslant \Gamma(t, x)+\varepsilon \\
\text { for a.e. }(t, x) \in J \text { and all } u \in R . \\
|f(t, x, u)| \leqslant \alpha(t, x)
\end{gathered}
$$

for some $\alpha \in H$ only depending on $\gamma, \Gamma, c$ and $d$. In order to apply coincidence degree (see e.g. [10] p. 44) we consider the following homotopy :

$$
u_{t}(t, x)-u_{x x}(t, x)=(1-\lambda) \Gamma(t, x) u(t, x)+\lambda \tilde{\gamma}(t, x, u(t, x)) u(t, x)+
$$

$$
\lambda f(t, x, u(t, x))+\lambda h(t, x) \quad(t, x) \in J
$$

where $\lambda \in(0,1)$ and $u \in$ Dom $L \quad(L$ as defined in Section 0$)$.

We have to show that the set of all possible solutions of the equation (1.12) is bounded independently of $\lambda \in(0,1)$. By construction, we have, for all $u \in \operatorname{Dom} L, \gamma(t, x)-\varepsilon \leqslant(1-\lambda) \Gamma(t, x)+\lambda \tilde{\gamma}(t, x, u(t, x))$ $\leqslant \Gamma(t, x)+\varepsilon$ for a.e. $(t, x) \in J$ and hence by Lemma 1.2 , one has

$$
|L u-[(1-\lambda) \Gamma(., .) u+\lambda \tilde{\gamma}(., ., u) u]| \geqslant \delta|u|_{1}
$$

for each $u \in \operatorname{Dom} L$ and each $\lambda \in(0,1)$. 
Consequently, from (1.11) one has

$$
|L u-[(1-\lambda) \Gamma(., .) u+\lambda \tilde{\gamma}(., ., u) u+\lambda f(., ., u)+\lambda h(., .)]| \geqslant \delta|u|_{1}-|e|
$$

for $u \subseteq \operatorname{Dom} L, \lambda \in(0,1)$ where $e=f+h$.

If we define the following operators

$$
\begin{aligned}
& A: H \rightarrow H, u+\Gamma(., .) u \\
& N: H \rightarrow H, u \rightarrow \tilde{\gamma}(., ., u) u+f(., ., u)+h(., .)
\end{aligned}
$$

then, $A$ is linear, $L$-completely continuous, $\operatorname{Ker}(L-A)=\{0\}$ from Lemma 1.2 and by our assumptions on $g, N$ is continuous and takes bounded sets into bounded sets, and hence $L$-completely continuous $[10]$. Therefore, if $u \in$ Dom $L$ is a solution of (1.12), it follows from (1.13) that

$|u|_{1} \leqslant \frac{|e|}{\delta}$. Thus from Theorem IV.5 in [10] there exists at least one solution for the equation $(H)$ and the proof is complete.

THEOREM 2. Assume that the inequalities

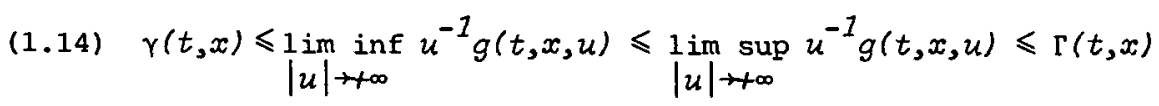

hold uniformly for a.e. $(t, x) \in J$, where $y \in L^{\infty}(J)$ and $\Gamma \in L^{\infty}(J)$ satisfies the following conditions:

(1.15) $\left\{\begin{array}{r}\Gamma(t, x) \leqslant 1 \text { for a.e. }(t, x) \in J \text { and } \\ b(t) \equiv \int_{0}^{\pi}(1-\Gamma(t, x)) \sin ^{2} x d x>0 \text { for a.e. } t \in[0,2 \pi] .\end{array}\right.$

Then the probiem (H) has at least one GPS for each $h \in H$.

LEMMA 1.3. Let $p \in L^{\infty}(J)$ be such that $p(t, x) \leqslant 1$ for a.e. $(t, x) \in J$ and $\int_{0}^{\pi}(1-p(t, x)) \sin ^{2} x d x>0$ for a.e. $t \in[0,2 \pi]$ then the. equation (1.5) has only the trivial solution. $u \in H_{o}^{1}(J)$,

Proof. It follows from Parseval-steklov equality that for any

$$
\left(u_{x}, u_{x}\right) \geqslant(u, u)
$$


with equality if and only if $u(t, x)=\sum_{k \in \mathbb{Z}} u_{k} e^{i k t} \sin x$. Therefore, if $u$ is a solution of (1.5), then

$$
0=(L u-p u \cdot u)=\left(u_{x}, u_{x}\right)-(p u, u) \geqslant 0
$$

and $u(t, x)=\sin x \sum_{k \in \mathbb{Z}} u_{k} e^{i k t} \equiv \sin x \cdot v(t)$ so that, by (1.17), one has

$$
\int_{0}^{2 \pi} \int_{0}^{\pi}(1-p(t, x)) \sin ^{2} x d x(v(t))^{2} d t=0 \text { and }
$$

from our assumptions, one must have $v(t)=0$ for a.e. $t \in[0,2 \pi]$ and the proof is complete.

Proof of Theorem 2. Using notations, the approach of Theorem 1 and Lemma 1.3 (instead of Lemma 1.1) one gets the conclusion and the proof is complete.

REMARK 2. It is obvious that the equation

$$
u_{t}(t, x)-u_{x x}(t, x)=(\cos x) u(t, x)
$$

satisfies conditions of Lemma 1.3.

REMARK 3. Similar results hold in the case of Periodic-Neuman boundary conditions and Periodic-Periodic boundary conditions if $[0, \pi]$ is replaced everywhere by $[0,2 \pi]$ in the last case.

REMARK 4. We have considered the period to be equal to $2 \pi$ only for the sake of commodity, one can consider any real number $T>0$.

\section{References}

[1] L. Amaral and M. P. Pera, "A note on periodic solutions at resonance", Bol. Un. Ital., to appear.

[2] H. Berestycki and D. G. De Figueiredo, "Double resonance in semilinear elliptic problems", Comm. in Partial Diff. Eq., 6(1) (1981), 91-120.

[3] H. Brezis and L. Nirenberg, "Characterizations of the range of some nonlinear operators and applications to boundary value problems", Ann. Scuola Norm. Sup. Pisa (4)5 (1978), 225-326. 
[4] D. G. De Figueiredo and J. P. Gossez, "Nonlinear perturbations of a linear elliptic problem near its first eigenvalue", J. Differential Eq. 30 (1978), 1-19.

[5] E. De Pascale and R. Iannacc1, "Periodic solutions of generalized Lienard equations with delay", to appear.

[6] S. Fucik, Solvability of nonlinear equations and boundary value problems (Reidel, Dordrecht, 1980).

[7] J. P. Gossez, "Some nonlinear differential equations with resonance at the first eigenvalue", Confer. semin. Math. Univ. Bari $n^{\circ} 167$ (1979), 355-389.

[8] R. Iannacci and M. N. Nkashama, "Nonresonance conditions for periodic solutions of forced Lienard and Duffing equations with delay", Rapport no. 29, Séminaire de Math (nouvelle série), Univ. de Louvain, mai 1983.

[9] J. Mawhin, "Periodic solutions of nonlinear telegraph equations" in Dynomical Systems, 193-210 (Bednarek and Cesari (eds), Academic Press, 1977).

[10] J. Mawhin, "Topological degree methods in nonlinear boundary value problems", Regional Conf. in Math no. 40, American Math. Soc., Providence, R.I., 1979.

[11] J. Mawhin, "Nonresonance conditions of nonuniform type in nonlinear boundary value problems", Dynamical Systems II , 255-276 (Academic Press, New York, 1982).

[12] J. Mawhin and J. R. Ward, Jr., "Nonresonance and existence for nonlinear elliptic boundary value problems", Nonlinear Anal. Theory, Methods, App I. 6 (1981), 677-684.

[13] J. Mawhin and J. R. Ward, Jr., "Nonuniform nonresonance conditions at the two first eigenvalues for periodic solutions of forced Lienard and Duffing equations", Rocky Mountain J. Math., 12(4) (1982), 643-654.

[14] J. Mawhin and J. R. Ward, Jr., "Asymptotic nonuniform conditions in the periodic-Dirichlet problem for semi-linear wave equations", to appear. 
[15] J. Mawhin, J. R. Ward, Jr. and M. Willem, "Variational methods and semi-linear elliptic equations", to appear.

[16] V. Stastnova and S. Fucik, "Weak periodic solutions of the boundary value problem for nonlinear heat equation", Aplikace matematiky 24 (1979), 284-303.

[17] V. Stastnova and S. Fucik, "Note to periodic solvability of the boundary value problem for nonlinear heat equation", Comment. Math. Univ. Carolinae 18 (1977), 735-740.

[18] 0 . Vejvoda, "Periodic solutions of nonlinear partial differential equations of evolution", in Differential Equations and their Applications, 293-300, Acta. Fac. Rer. Natur. Univ. Comenianae, Math. 17 (Bratislava, 1967).

[19] 0. Vejvoda et al., Partial differential equations : time-periodic solutions (Sijthoff Noordhoff, 1981).

Institut de Mathematique,

Université de Louvain,

Chemin du Cyclotron 2,

B-1348 Louvain-1a-Neuve,

Belgium. 